\title{
Nonparametric Bayesian Multiple Comparisons for Dependence Parameter in Bivariate Exponential Populations
}

\author{
M. Masoom Ali \\ Ball State University, mali@bsu.edu \\ J. S. Cho \\ Kyungsung University \\ Munni Begum \\ Ball State University
}

Follow this and additional works at: http://digitalcommons.wayne.edu/jmasm

Part of the Applied Statistics Commons, Social and Behavioral Sciences Commons, and the Statistical Theory Commons

\section{Recommended Citation}

Ali, M. Masoom; Cho, J. S.; and Begum, Munni (2006) "Nonparametric Bayesian Multiple Comparisons for Dependence Parameter in Bivariate Exponential Populations," Journal of Modern Applied Statistical Methods: Vol. 5 : Iss. 1 , Article 7.

DOI: $10.22237 /$ jmasm/1146456360

Available at: http://digitalcommons.wayne.edu/jmasm/vol5/iss1/7

This Regular Article is brought to you for free and open access by the Open Access Journals at DigitalCommons@WayneState. It has been accepted for inclusion in Journal of Modern Applied Statistical Methods by an authorized editor of DigitalCommons@WayneState. 


\section{Nonparametric Bayesian Multiple Comparisons for Dependence Parameter in Bivariate Exponential Populations}

\author{
M. Masoom Ali \\ Dept. of Math. Sciences \\ Ball State University
}

\author{
J. S. Cho \\ Dept. of Inform. Statistics \\ Kyungsung University
}

\author{
Munni Begum \\ Dept. of Math. Sciences \\ Ball State University
}

A nonparametric Bayesian multiple comparisons problem (MCP) for dependence parameters in I bivariate exponential populations is studied. A simple method for pairwise comparisons of these parameters is also suggested. The methodology by Gopalan and Berry (1998) is extended using Dirichlet process priors, applied in the form of baseline prior and likelihood combination to provide the comparisons. Computation of the posterior probabilities of all possible hypotheses are carried out through a Markov Chain Monte Carlo, Gibbs sampling, due to the intractability of analytic evaluation. The process of MCP for the dependent parameters of bivariate exponential populations is illustrated with a numerical example.

Key words: Bivariate exponential population; Dirichlet process prior; Gibbs sampler; mixture of Dirichlet processes; multiple comparison; nonparametric Bayes.

\section{Introduction}

In reliability studies of mechanical components, dependence between two components occurs quite often. A system, which functions as long as at least one of the two identical components functions, has a functional correlation between the system components. Initially, let the two components be independently on test with life distributions that are exponential with parameter $\lambda$, denoted as $\exp (\lambda)$. Failure of one changes the life distribution of the other to $\exp (\lambda \theta), \theta>0$. When $\theta=1$, the two components function independently. For $\theta>1$, the workload of the remaining component is increased, thereby decreasing the mean life. Here $\theta$ is

M. Masoom Ali is George \& Frances Ball Distinguished Professor of Statistics. Email him at mali@bsu.edu. His research interests are in order statistics, Bayesian statistics, statistical inference and distribution problems. J. C. Cho is Associate Professor of Informational Statistics. His research interests are in Bayesian Statistics. Munni Begum is Assistant Professor of Statistics. Her research interests are in Bayesian Statistics, Statistical modeling and Biostatistical methods. called the dependence parameter. Weier (1981) provided the Bayes estimators of the parameters and reliability using a conjugate prior for such problems.

The multiple comparison problem (MCP) for $I$ bivariate exponential populations with dependence parameters $\boldsymbol{\theta}=\left(\theta_{1}, \ldots \ldots, \theta_{I}\right)$ can be viewed as making inferences concerning relationships among the $\theta$ 's based on observations. This is tantamount to testing the following hypothesis,

$$
H_{0}: \theta_{1}=\ldots \ldots . .=\theta_{I} \text { vs. } H_{l}: \text { not } H_{0} .
$$

For bivariate exponential populations, the frequentist approach of multiple comparison is not very straightforward. This is partly due to the difficulty in handling the distributional aspects and associated computations. The multiple comparison problem using nonparametric priors in a Bayesian inferential setup was studied by Gopalan and Berry (1998) providing specific applications to the Binomial and Normal populations. Following similar approach, the MCP for a set of geometric and negative binomial populations (Masoom, Cho, \& Begum, 2005) was studied. In this article, the MCP for the dependence parameters of a set of 
bivariate exponential populations along the same line was studied.

In a Bayesian approach, the posterior probabilities of respective hypotheses in MCP can be calculated with moderate effort. The prior information on the unknown parameters has to be quantified as a distribution. However, the selection of the prior distribution could be tricky. One of the criticisms Bayesian inferential methods often face is the subjectivity in prior specification. In real data analysis prior specification could be based on scientific knowledge about the parameters. Noninformative prior specification is optimal in cases when there is little known about the background information. It is very important that prior distributions be as objective as possible while doing Bayesian inference. A typical objective prior distribution is the Dirichlet process prior (DPP) that leads to nonparametric Bayesian inference.

The DPP is a prior distribution on the family of distributions that is dense in the space of distribution functions. The family of DPPs was introduced by Ferguson (1973) and was extended to mixtures of DPP by Antoniak (1974) in order to treat problems including the estimation of a mixing distribution, bio-assay, empirical Bayes problems and discrimination problems. Escobar (1988) started the application of Markov chain Monte Carlo (MCMC) methods in nonparametric Bayesian modeling. Novel computational techniques and developments of MCMC schemes, including key contributions by Doss (1994), Bush and MacEachern (1996), Escobar and West (1997), MacEachern and Müller (1998), West, Müller and Escobar (1994) made it possible to study nonparametric Bayesian methods widely.

The focus was on the Bayesian approach to the multiple comparisons problem for $I$ bivariate exponential populations based on the nonparametric Dirichlet process priors in this article. The MCMC techniques, in particular Gibbs sampling, is adopted here to evaluate the posterior probabilities of the hypotheses.

\section{Preliminaries}

Let $(X, Y)$ denote the lifetimes of the two components that have a bivariate exponential model. The joint probability density function of $(X, Y)$ can be written as,

$$
\begin{aligned}
& f(x, y) \mid \lambda, \theta)=2 \theta \lambda^{2} \exp (-2 \lambda x-\lambda \theta y), \\
& x, y>0, \quad \lambda, \theta>0
\end{aligned}
$$

with $\theta$ as the dependence parameter.

It is assumed that $(\boldsymbol{x}, \boldsymbol{y})=\left\{\left(\boldsymbol{x}_{1}, \boldsymbol{y}_{1}\right),\left(\boldsymbol{x}_{2}\right.\right.$, $\left.\left.\boldsymbol{y}_{2}\right), \ldots . .,\left(\boldsymbol{x}_{\mathrm{I}}, \boldsymbol{y}_{\mathrm{I}}\right)\right\}$ be a set of observations available on $I$ populations, where $\left(\boldsymbol{x}_{\mathrm{i}}\right.$, $\left.\boldsymbol{y}_{\mathrm{i}}\right)=\left\{\left(x_{i 1}, y_{i l}\right), \ldots \ldots,\left(x_{i n i}, y_{i n i}\right)\right\}$ is an $n_{i} \times 1$ vector of conditionally independent observations on population $i, i=1,2, \ldots . ., I ; j=1,2, \ldots . ., n_{i}$ and $\sum_{i=1}^{I} n_{i}=n$. Then the probability density function of $\left(x_{i j}, y_{i j}\right)$ is,

$$
\begin{aligned}
& f\left(x_{i j}, y_{i j} \mid \lambda_{i}, \theta_{i}\right)=2 \theta_{i} \lambda_{i}^{2} \times \exp \left(-2 \lambda_{i} x_{i j}-\lambda_{i} \theta_{i} y_{i j}\right), \\
& x_{i j}, y_{i j}>0, \quad \lambda_{i}, \theta_{i}>0 .
\end{aligned}
$$

Now a distribution function $G_{0}($.$) and a$ positive scalar precision parameter $\alpha$ together determine the Dirichlet process prior $G$. Here $G_{0}$ (.) that defines the location of the DPP is sometimes called prior guess or baseline prior. The precision parameter $\alpha$ determines the concentration of the prior for $G$ around the prior guess $G_{0}$, and therefore measures the strength of belief in $G_{0}$. The DPP is usually denoted by $G \sim$ $D\left(G \mid G_{0}, \alpha\right)$. For large values of $\alpha, G$ is very likely to be close to $G_{0}$, while for small values of $\alpha, G$ is likely to put most of its probability mass on just a few atoms.

It is assumed that the $\theta_{i}$ 's come from $G$, and that $G \sim D\left(G \mid G_{0}, \alpha\right)$ as stated above. This structure results in a posterior distribution which is a mixture of Dirichlet processes (Antoniak 1974). Now following the Polya urn representation of the Dirichlet process (Blackwell \& MacQueen, 1973), the joint posterior distribution can be written as, 


$$
\theta_{i} \mid \mathbf{x}, \mathbf{y} \propto \prod_{i=1}^{I} f\left(\mathbf{x}_{i}, \mathbf{y}_{i} \mid \theta_{i}\right) \times \frac{\alpha G_{0}\left(\theta_{i}\right)+\sum_{k<i} \delta\left(\theta_{i} \mid \theta_{k}\right)}{\alpha+i-1}
$$

where $\delta\left(\theta_{i} \mid \theta_{k}\right)$ is the distribution putting a point mass on $\theta_{k}$. For each $i=1, \ldots \ldots I$, the conditional posterior distribution of $\theta_{i}$ is given by,

$$
\begin{aligned}
& \theta_{i} \mid \theta_{k}, k \neq i, \mathbf{x}, \mathbf{y} \propto q_{0} G_{b}\left(\theta_{i} \mid \mathbf{x}_{i}, \mathbf{y}_{i}\right)+ \\
& \sum_{k \neq i} q_{k} \delta\left(\theta_{i} \mid \theta_{k}\right),
\end{aligned}
$$

where $G_{b}\left(\theta_{\mathrm{i}} \mid \boldsymbol{x}_{\mathrm{i}}, \boldsymbol{y}_{\mathrm{i}}\right)$ is the baseline posterior distribution, $\quad q_{0} \propto \alpha \int f\left(\mathbf{x}_{i}, \mathbf{y}_{i} \mid \theta\right) d G_{0}\left(\theta_{i}\right)$, $q_{k} \propto f\left(\mathbf{x}_{i}, \mathbf{y}_{i} \mid \theta_{k}\right)$, and $1=q_{0}+\sum_{k \neq i} q_{k}$. Let $\boldsymbol{\Theta}=\left\{\boldsymbol{\theta}=\left(\theta_{1}, \theta_{2}, \ldots \ldots, \theta_{I}\right): \theta_{i} \in R, i=1,2, \ldots \ldots\right.$, $I\}$ be the $I$-dimensional parameter space. Equality and inequality relationships among $\theta$ 's induce statistical hypotheses that are subsets of $\boldsymbol{\Theta}$. Thus, the MCP becomes testing the following hypotheses.

$H_{0}: \boldsymbol{\theta}_{0}=\left\{\theta_{i}: \theta_{1}=\theta_{2}=\ldots \ldots=\theta_{I}\right\}$,

$H_{1}: \boldsymbol{\theta}_{1}=\left\{\theta i: \theta_{1} \neq \theta_{2}, \quad \theta_{2}=\theta_{3}=\ldots \ldots=\right.$

$\left.\theta_{I}\right\}, \ldots \ldots . H_{N}: \boldsymbol{\theta}_{N}=\left\{\theta_{i}: \theta_{1} \neq \theta_{2} \neq \theta_{3} \neq \ldots \ldots \neq \theta_{K}\right\}$.

The hypotheses $H_{r}: \boldsymbol{\theta}_{r}, r=0,1,2, \ldots \ldots ., N$, are disjoint, and $\bigcup_{r=0}^{n} \theta_{r}=\Theta$.

The elements of $\boldsymbol{\Theta}$ themselves behave as described by (3) and so with positive probability, they will reduce to some $p<I$ distinct values. Let superscript $*$ denote distinct values of the parameters. Then, any realization of $I$ parameters $\theta_{\mathrm{i}}$ generated from $G$ lies in a set of $p<I$ distinct values, denoted by $\left(\boldsymbol{\theta}^{*}=\theta_{1}{ }^{*}, \theta_{2}{ }^{*}\right.$, ....., $\left.\theta_{p}^{*}\right)$. The computation of posterior probabilities for different hypotheses through Gibbs algorithm becomes manageable using the notion of configuration as termed by Gopalan and Berry (1998). Their definition of configuration is restated here:
Definition (Configuration): The set of indices $S$ $=\left\{S_{1}, \ldots ., S_{I}\right\}$ determines a classification of the data $\boldsymbol{\Theta}=\left\{\theta_{1}, \ldots \ldots, \theta_{I}\right\}$ into $I^{*}$ distinct groups or clusters; the $n_{j}=\#\left\{S_{i=j}\right\}$ observations in group $j$ share the common parameter value $\theta_{\mathrm{j}}^{*}$. Now, define $I_{j}$ as the set of indices of observations in group $j$; That is, $I_{j}=\left\{i: S_{i}=j\right\}$. Let $(X, Y)_{(j)}=$ $\left\{\left(X_{i}, Y_{i}\right): S_{i}=j\right\}$ be the corresponding group of $n_{I_{j}}=\sum_{i \in I_{j}} n_{i}$ observations. Thus, a one-to-one

correspondence between hypotheses and configurations follows and the required computations are reduced by the fact that the distinct $\theta_{\mathrm{i}}$ 's are typically reduced to fewer than $I$ due to the clustering of the $\theta_{i}$ 's inherent in the Dirichlet process. Hence, (4) can be rewritten as:

$$
\begin{aligned}
& \theta_{i} \mid \theta_{k}, k \neq i, \mathbf{x}, \mathbf{y} \propto q_{0} G_{b}\left(\theta_{i} \mid \mathbf{x}_{i}, \mathbf{y}_{i}\right)+ \\
& \sum n_{k} q_{k}^{*} \delta\left(\theta_{i} \mid \theta_{k}^{*}\right),
\end{aligned}
$$

with $q_{k}^{*} \propto f\left(\mathbf{x}_{i}, \mathbf{y}_{i} \mid \theta^{*}{ }_{k}\right)$, and $1=q_{0}+\sum_{k \neq i} n_{k} q_{k}^{*}$. In addition to the simplification of notations, the cluster structure of the $\theta_{\mathrm{i}}$ also improves the efficiency of the algorithm.

Posterior Sampling In Dirichlet Process Mixtures

A gamma distribution with parameters $\left(\alpha_{0 i}, \beta_{0 i}\right)$ is considered as baseline prior $G_{0}$. This implies that $\theta_{1}, \theta_{2}, \ldots \ldots, \theta_{I}$ are i.i.d. from $G_{0}$. Then, a hierarchical set up for the Dirichlet process analysis as outlined above becomes,

$$
\begin{gathered}
\mathbf{x}_{i}, \mathbf{y}_{i} \mid \theta_{i} \sim \operatorname{BVE}\left(\mathbf{x}_{i}, \mathbf{y}_{i} \mid \lambda_{i}, \theta_{i}\right), \\
\theta_{i} \mid G \sim G\left(\theta_{i}\right), \\
G \mid G_{0}, \alpha \sim D\left(G \mid G_{0}, \alpha\right), \\
G_{0} \mid \alpha_{0 i}, \beta_{0 i} \sim \operatorname{Gam}\left(\alpha_{0 i}, \beta_{0 i}\right),
\end{gathered}
$$




$$
\lambda_{i} \mid \alpha_{1 i}, \beta_{1 i} \sim \operatorname{Gam}\left(\alpha_{1 i}, \beta_{1 i}\right)
$$

$B V E$ and Gam stand for bivariate exponential and gamma distributions, respectively. Now, the choice of the precision parameter $\alpha$ in Dirichlet process is extremely important for the model. A gamma prior for $\alpha$ with a shape parameter $a$ and scale parameter $b$ is considered, that is, $\alpha \sim$ $\operatorname{Gam}(a, b)$. Thus, the $\operatorname{Gam}(a, b)$ becomes the reference prior if $a \rightarrow 0$ and $\mathrm{b} \rightarrow 0$ and one has access to a neat data augmentation device for sampling $\alpha$ by Escobar and West (1995).

The configuration notation is more convenient to use in describing the Gibbs sampling algorithm as the full conditionals can be written in closed form as under:

$$
\begin{gathered}
\left(\theta_{i} \mid \mathbf{x}, \mathbf{y}, \theta_{k}, k \neq i, \alpha\right) \sim \\
q_{0} \operatorname{Gam}\left(n_{i}+\alpha_{0 i}, \lambda_{i} \sum_{j=1}^{n_{i}} y_{i j}+\beta_{0 i}\right)+\sum_{k \neq i} q_{k} \delta\left(d \theta_{i} \mid \theta_{k}\right),
\end{gathered}
$$

$$
\begin{gathered}
\left(\lambda_{i} \mid \mathbf{x}, \mathbf{y}, \theta_{i}, \alpha\right) \sim \\
\operatorname{Gam}\left(2 n_{i}+\alpha_{1 i}, 2 \sum_{j=1}^{n_{i}} x_{i j}+\theta_{i} \sum_{j=1}^{n_{i}} y_{i j}+\beta_{1 i}\right)
\end{gathered}
$$

$$
\begin{gathered}
\left(\theta_{j}^{*} \mid \mathbf{x}, \mathbf{y}, \mathrm{S}\right) \sim \\
\operatorname{Gam}\left(\sum_{i=1}^{I^{*}} n_{i}+\alpha_{0 i}^{*}, \lambda_{i} \sum_{i=1}^{I^{*}} \sum_{j=1}^{n_{i}} y_{i j}+\beta_{0 j}^{*}\right),
\end{gathered}
$$

$$
\begin{gathered}
\left(\alpha \mid \eta, I^{*}\right) \sim \\
\pi_{\eta} \operatorname{Gam}\left(a+I^{*}, b-\log (\eta)\right)+ \\
\left(1-\pi_{\eta}\right) \operatorname{Gam}\left(a+I^{*}-1, b-\log (\eta)\right),
\end{gathered}
$$

$$
\left(\eta \mid \alpha, I^{*}\right) \sim \operatorname{Beta}\left(\alpha+1, I^{*}\right)
$$

where

$$
\begin{gathered}
q_{0} \propto \alpha \lambda_{i}^{2 n_{i}+\alpha_{0 i}-1} \exp \left(-2 \lambda_{i} \sum_{j=1}^{n_{i}} x_{i j}\right) \\
\frac{\Gamma\left(n_{i}+\alpha_{0 i}\right)}{\left[\lambda_{i}\left(\sum_{j=1}^{n_{i}} y_{i j}+\beta_{0 i}\right)\right]^{n_{i}+\alpha_{0 i}}}, \\
q_{k} \propto \theta_{k}^{n_{i}} \lambda_{k}^{2 n_{i}} \exp \left(-2 \lambda_{k} \sum_{j=1}^{n_{i}} x_{i j}-\theta_{k} \lambda_{k} \sum_{j=1}^{n_{i}} y_{i j}\right)
\end{gathered}
$$

Gibbs sampling proceeds by simply iterating through (11) - (15) in order, sampling at each stage based on the current values of all the conditioning variables.

The configuration induces the equality and inequality relationships among the $\theta$ 's that corresponds to the partitions on the parameter space $\boldsymbol{\Theta}$ and in turn to the hypotheses of interest. In order to estimate the posterior probability of a hypothesis $H_{r}$ from a large number $(L)$ of sample draws, one takes

$$
P\left(H_{r} \mid \mathbf{X}, \mathbf{Y}\right) \approx \frac{1}{L} \sum_{l=1}^{L} \delta_{S_{l}}\left(H_{r}\right)
$$

where $\delta_{S_{l}}\left(H_{r}\right)$ denotes unit point mass for the case where $l$ th draw of $S, S_{0}$ corresponds to $H_{r}$. The probability of equality for any two $\theta$ 's can be calculated from the posterior distributions on hypotheses, $P\left(H_{r} \mid \boldsymbol{X}, \boldsymbol{Y}\right), \quad r=1,2, \ldots \ldots ., N$. This can be achieved by adding probabilities of those hypotheses in which the two $\theta_{\mathrm{i}}$ and $\theta_{\mathrm{j}}$ are equal. That is

$$
\begin{array}{r}
P\left(\theta_{i}=\theta_{j} \mid \mathbf{X}, \mathbf{Y}\right) \approx \frac{1}{L} \sum_{l=1}^{L} \delta_{S_{l}}\left(\theta_{i}=\theta_{j}\right)= \\
\sum_{r=1}^{N} P\left(H_{r} \mid \mathbf{X}, \mathbf{Y}\right) \delta_{H_{r}}\left(\theta_{i}-\theta_{j}\right), i \neq j,
\end{array}
$$

where $\delta_{S_{l}}\left(\theta_{i}-\theta_{j}\right)$ and $\delta_{H_{r}}\left(\theta_{i}=\theta_{j}\right)$ denote unit point mass for the case where $S_{l}$ and $H_{r}$ indicate $\theta_{i}=\theta_{j}$. 
Illustrative Example

A numerical illustration of the multiple comparisons for the dependence parameters in bivariate exponential populations is presented in this section using simulated data. Four bivariate exponential populations each with size $n_{i}=20$ are considered. Then, the numbers of possible hypotheses for multiple comparisons are 15 . The observed summary statistics for these data are given in Table 1.

It follows from Table 1 , that the true hypothesis may be $H_{\text {true }}$ : $\theta_{1}=\theta_{2} \neq \theta_{3}=\theta_{4}$. For the precision parameter $\alpha$, one considers three Gamma priors with parameters $(a, b)=(1.0,1.0)$, $(0.1,0.1)$ and $(0.01,0.01)$ in order to have equal mean 1 and different variances 1, 10, and 100, respectively. This also facilitates that the latter prior be fairly non-informative, giving reasonable mass to both high and low values of $\alpha$. As well, each $\theta_{i}, \mathrm{i}=1, \ldots \ldots, 4$ were set $a$ priori following a gamma distribution with parameters $\alpha_{0 i}=\alpha_{1 i}=2.0$ and $\beta_{0 i}=\beta_{1 i}=0.001$ to reflect vagueness of the prior knowledge.

The posterior probabilities for all possible hypotheses are approximated by the Gibbs sampling algorithm using 20,000 iterations with 10,000 burn-ins and 5 replications and are presented in Table 2. It is to be noted that the hypothesis $\theta_{1}=\theta_{2} \neq \theta_{3}=\theta_{4}$ has the largest posterior probabilities $0.7883,0.7274$ and 0.7410 for all priors of the precision parameter $\alpha$. Thus, the data lend greatest support to equalities for $\theta_{1}=\theta_{2}$ and $\theta_{3}=\theta_{4}$ being different from the others.

Table 3 presents the pairwise posterior probabilities for the equalities in pairs of $\theta$ 's. The equalities of $\left(\theta_{1}=\theta_{2}\right)$ and $\left(\theta_{3}=\theta_{4}\right)$ have the largest posterior probabilities $(0.9943,0.9903$, $0.9729)$ and $(1.0000,1.0000,1.0000)$ for three cases of $(a, b)$ respectively. This suggests that there is strong evidence in the equality $\left(\theta_{1}=\theta_{2}\right)$ and $\left(\theta_{3}=\theta_{4}\right)$.

The Bayesian approach using nonparametric Dirichlet process priors facilitates studying the problem of multiple comparisons in a number of different distributions. So far, the MCP was carried out for a univariate distribution. Here, it has been shown that the method can be extended to a bivariate distribution as well, with moderate effort. As an alternative to a formal Bayesian analysis of a mixture model that usually leads to intractable calculations, the DPP is used to provide a nonparametric Bayesian method for obtaining posterior probabilities for various hypotheses of equality among the dependence parameters of bivariate exponential populations.

Table 1 The observed summary statistics for each populations

$\begin{array}{ccccc}\frac{\text { Populations }}{X_{i}=\sum_{j=1}^{n_{i}} X_{i j}} & 1.500 & 1.560 & 0.700 & 0.720 \\ Y_{i}=\sum_{j=1}^{n_{i}} Y_{i j} & 6.500 & 6.000 & 1.300 & 1.130 \\ \hat{\theta}_{M L E} & 0.462 & 0.520 & 1.077 & 1.274\end{array}$


Table 2 Calculated posterior probabilities for each hypothesis with three cases of $(\mathrm{a}, \mathrm{b})$

$\begin{array}{cccc}\text { Hypothesis } & (1.0,1.0) & (0.1,0.1) & (0.01,0.01) \\ \theta 1=\theta 2=\theta 3=\theta 4 & .2059 & .2629 & .2320 \\ \theta 1=\theta 2=\theta 3 \neq \theta 4 & .0000 & .0000 & .0000 \\ \theta 1=\theta 2=\theta 4 \neq \theta 3 & .0000 & .0000 & .0000 \\ \theta 1=\theta 2 \neq \theta 3=\theta 4 & .7883 & .7274 & .7410 \\ \theta 1=\theta 2 \neq \theta 3 \neq \theta 4 & .0000 & .0000 & .0000 \\ \theta 1=\theta 3=\theta 4 \neq \theta 2 & .0036 & .0038 & .0030 \\ \theta 1=\theta 3 \neq \theta 2=\theta 4 & .0000 & .0000 & .0000 \\ \theta 1=\theta 3 \neq \theta 2 \neq \theta 4 & .0000 & .0000 & .0000 \\ \theta 1=\theta 4 \neq \theta 2=\theta 3 & .0000 & .0000 & .0000 \\ \theta 1=\theta 4 \neq \theta 2 \neq \theta 3 & .0000 & .0000 & .0000 \\ \theta 1 \neq \theta 2=\theta 3=\theta 4 & .0003 & .0007 & .0015 \\ \theta 1 \neq \theta 2=\theta 3 \neq \theta 4 & .0000 & .0000 & .0000 \\ \theta 1 \neq \theta 2=\theta 4 \neq \theta 3 & .0000 & .0000 & .0000 \\ \theta 1 \neq \theta 2 \neq \theta 3=\theta 4 & .0018 & .0052 & .0226 \\ \theta 1 \neq \theta 2 \neq \theta 3 \neq \theta 4 & .0000 & .0000 & .0000\end{array}$

Table 3 Pairwise Posterior Probabilities with three cases of $(a, b)$

$\begin{array}{cccc}\text { Hypothesis } & (1.0,1.0) & (0.1,0.1) & (0.01,0.01) \\ \theta_{1}=\theta_{2} & .9943 & .9903 & .9729 \\ \theta_{1}=\theta_{3} & .2096 & .2667 & .2349 \\ \theta_{1}=\theta_{4} & .2096 & .2667 & .2349 \\ \theta_{2}=\theta_{3} & .2062 & .2636 & .2334 \\ \theta_{2}=\theta_{4} & .2062 & .2636 & .2334 \\ \theta_{3}=\theta_{4} & 1.0000 & 1.0000 & 1.0000\end{array}$

References

Ali, M. Masoom, Cho, J. S., Begum, M. (2005). Nonparametric Bayesian multiple comparisons for geometric populations. Journal of the Korean Data and Information Science Society, 16(4), 1129-1140.

Ali, M. Masoom., Cho, J. S., Begum, M. (2006). Bayesian multiple comparisons with nonparametric dirichlet process priors for negative binomial populations. Pakistan Journal of Statistics, 22(2), 88-98.
Antoniak, C. E. (1974). Mixtures of dirichlet processes with applications to nonparametric problems. The Annals of Statistics, 2, 1152-1174.

Bush, C. A. \& MacEachern, S. N. (1996). A semi-parametric bayesian model for randomized block designs. Biometrika, 83, 275285.

Doss, H. (1994). Bayesian nonparametric estimation for incomplete data via successive substitution sampling. The Annals of Statistics, 22, 1763-1786. 
Escobar, M. D. (1988). Estimating the means of several normal populations by nonparametric estimation of the distribution of the means. Unpublished dissertation, Yale University.

Escobar, M. D. \& West, M. (1997). Computing nonparametric hierarchical models. ISDS Discussion Paper \#97-15, Duke University.

Ferguson, T. S. (1973). A bayesian analysis of some nonparametric problems. The Annals of Statistics, 1, 209-230.
Gopalan, R. \& Berry, D. A. (1998). Bayesian multiple comparisons using dirichlet process priors. Journal of the American Statistical Association, 90, 1130 - 1139.

MacEachern, S. N. \& Müller, P. (1998). Estimating mixture of dirichlet process models. Journal of Computational and Graphical Statistics, 7, 223-239.

Weier, D. R. (1981). Bayes estimation for a bivariate survival model based on exponential distributions. Communication in Statistics-Theory and Methods, 10, 1415-1427. 